\title{
Eficiência de Uso dA Água em Genótipos de CANA-DE-Açúcar Submetidos À AplicaçÃo de Herbicidas ${ }^{1}$
}

\author{
Water Use Efficiency in Sugarcane Genotypes Submitted to Herbicide Application
}

\author{
GALON, L. ${ }^{2}$, CONCENÇO, G. ${ }^{3}$, FERREIRA, E.A. ${ }^{3}$, ASPIAZÚ, I. ${ }^{3}$, SILVA, A.F. ${ }^{3}$, FERREIRA, F.A. ${ }^{4}$, \\ SILVA, A.A. ${ }^{4}$, TIRONI, S.P. ${ }^{3}$, FREITAS, M.A.M. ${ }^{5}$ e SOARES, E.R. ${ }^{6}$
}

\begin{abstract}
RESUMO - Objetivou-se com este trabalho avaliar os efeitos de ametryn e trifloxysulfuronsodium, em aplicações isoladas ou em mistura, sobre as características associadas à eficiência do uso da água em genótipos de cana-de-açúcar. O delineamento experimental foi em blocos casualizados, com quatro repetições, em parcelas subdivididas. As parcelas foram compostas pelos herbicidas ametryn e trifloxysulfuron-sodium e pela mistura formulada de ametryn + trifloxysulfuron-sodium, aplicados aos 65 dias após o plantio, além de uma testemunha sem presença de plantas daninhas; e as subparcelas, pelos genótipos RB72454, RB835486, RB855113, RB867515, RB947520 e SP80-1816. Decorridos 15 dias da aplicação dos herbicidas, foram realizadas as avaliações de condutância estomática de vapores de água (Gs), gradiente entre temperatura da folha e do ar $(\Delta \mathrm{T})$ e taxa de transpiração $(\mathrm{E})$, sendo calculada ainda a eficiência do uso da água (WUE). Foi determinada também a matéria seca da parte aérea das plantas. O genótipo RB855113 foi o que apresentou maiores danos à eficiência do uso da água e transpiração, sendo, por isso, considerado o mais sensivel aos herbicidas ametryn e trifloxysulfuron-sodium, enquanto os genótipos SP80-1816 e RB867515 estiveram entre os mais tolerantes. Condutância estomática, temperatura da folha, transpiração e eficiência de uso da água são características eficientes para identificar danos de herbicidas a plantas cultivadas, principalmente aqueles com efeito sobre a taxa fotossintética. Os genótipos de cana-de-açúcar avaliados diferiram quanto à sensibilidade aos herbicidas aplicados; as variedades SP80-1816 e RB867515 destacaram-se como as menos afetadas pelo ametryn e trifloxysulfuron-sodium, isolados ou em mistura, enquanto RB855113 foi a mais sensivel.
\end{abstract}

Palavras-chave: Saccharum spp., uso eficiente da água, mistura herbicida, cultivares de cana-de-açúcar.

\begin{abstract}
The objective of this study was to evaluate the effects of the herbicides ametryn and trifloxysulfuron-sodium, applied alone or in mixture, on the characteristics associated to water use efficiency in sugarcane varieties under field conditions. The trial was installed in a completely randomized block design with split-plots and four replications. Plots were composed by the herbicides ametryn, trifloxysulfuron-sodium or a commercial mixture of ametryn + trifloxysulfuron-sodium, applied 65 days after planting, plus a control mechanically free of weed infestation; split-plots were composed by sugarcane varieties (RB72454, RB835486, RB8551 13, RB867515, RB94752O and SP80-1816). Fifteen days after herbicide application, stomatal conductance (Gs), temperature gradient between leaf and air (DT) and transpiration rate (E) were evaluated, and water use efficiency (WUE) was obtained as a function of photosynthesis and transpiration rates. Plant shoots were also collected for dry matter determination. Variety RB855113 presented the greatest damage to wateruse efficiency and transpiration and was thus considered the most sensitive to ametryn and trifloxysulfuron, while varieties SP80-1816 and RB867515 were the most tolerant. In these varieties, herbicides caused only minor changes in wateruse efficiency and thermal gradient. Stomatal conductance, leaftemperature, transpiration and water use efficiency were effective in identifying herbicide damage to crops, mainly those caused by photosynthesis-inhibiting herbicides; sugarcane genotypes showed a different behavior in relation to herbicide susceptibility and varieties SP80-1816 and RB867515 were the less affected by the herbicide treatments; on the other hand, RB855113 was the most severely affected.
\end{abstract}

Keywords: Saccharum spp., efficient water use, herbicide mixture, sugarcane cultivars.

Recebido para publicação em 25.11.2009 e na forma revisada em 12.11.2010

2 Engô-Agr. Prof. da Universidade Federal do Pampa - UNIPAMPA, Campus Itaqui-RS, Rua Luiz de Joaquim de Sá Britto, s/n, Bairro Promorar, 97650-000 Itaqui-RS, <galonleandro@ig.com.br>; ${ }^{3}$ Engo-Agr ${ }^{0}$., Doutorando no Dep. de Fitotecnia, Universidade Federal de Viçosa - DFT/UFV; ${ }^{4}$ Eng - -Agr ${ }^{0}$., D.Sc., Professor do Dep. de Fitotecnia, DFT/UFV; ${ }^{5}$ Aluno do curso de Agronomia, UFV; ${ }^{6}$ Aluno do curso de Agronomia, FAMA.

Planta Daninha, Viçosa-MG, v. 28, n. 4, p. 777-784, 2010 


\section{INTRODUÇÃO}

Embora a água seja abundante no globo terrestre, menos de 1\% dela é doce, portanto apta para consumo humano e utilização na agricultura. Por esse motivo, o desenvolvimento de técnicas que permitam o uso eficiente da água para essas finalidades é prioritário em regiões do mundo. No caso do Brasil, estudos sobre o uso eficiente da água por culturas que ocupam grandes áreas, como a cana-de-açúcar, que se constitui na melhor alternativa como matéria-prima para o combustivel etanol (Andreoli \& Souza, 2006), tornaram-se de grande valia. A eficiência do uso da água é medida pela relação entre a quantidade de água evapotranspirada por uma cultura e sua produção de matéria seca (Concenço et al., 2007a, 2009). Assim, culturas mais eficientes no uso da água produzem mais matéria seca por grama de água transpirada (Baptista et al., 2001; Procópio et al., 2004). O uso mais eficiente da água está diretamente correlacionado ao tempo de abertura estomática, pois, enquanto a planta absorve $\mathrm{CO}_{2}$ para a fotossintese, a água é perdida por transpiração, com intensidade variável, dependendo do gradiente de potencial entre a superfície foliar e a atmosfera, seguindo uma corrente de potenciais hidricos (Pereira-Netto et al., 2002).

Acredita-se que alguns herbicidas possam influenciar a eficiência do uso da água pelo efeito negativo direto sobre a fotossintese, ou indiretamente, pela redução da taxa metabólica da planta. Contudo, especula-se a existência de diferenças marcantes entre os materiais genéticos de uma mesma espécie, que podem ser avaliadas quanto a diferenças na condutância estomática, taxa transpiratória, temperatura da folha e acúmulo de massa seca quando eles são tratados com herbicidas. Dessa forma, a associação genótipo/ herbicida pode determinar aqueles que sobressaem sob estresse hídrico quando da aplicação do produto e, assim, garantir melhores produções em nível de lavoura.

Dentre os herbicidas mais empregados para o controle de plantas daninhas em canade-açúcar, destacam-se o ametryn e o trifloxysulfuron-sodium, que controlam mono e dicotiledôneas, apresentando baixa toxicidade para animais e humanos (Vivian et al., 2007). $\mathrm{O}$ ametryn pertence ao grupo químico dos inibidores do fotossistema II; em função disso, seus danos à cultura podem ser aferidos pela mensuração direta da taxa fotossintética e de variáveis associadas a ela (Rodrigues \& Almeida, 2005). Já o trifloxysulfuron-sodium está incluído no grupo químico dos inibidores da enzima acetolactato sintase (ALS), que participa da biossintese dos aminoácidos de cadeia ramificada valina, leucina e isoleucina (Rodrigues \& Almeida, 2005). Embora não atue diretamente na rota fotossintética, ocasiona falência gradativa do metabolismo do vegetal, e seus danos às plantas da cultura podem ser avaliados pela sua influência indireta sobre a fotossintese e sobre as variáveis associadas a ela (Taiz \& Zeiger, 2006).

Desde 2001 utiliza-se o ametryn em mistura comercial com trifloxysulfuronsodium (Rodrigues \& Almeida, 2005), a qual apresenta fácil absorção pelo sistema de raízes e pelas folhas. Os sintomas de intoxicação ocasionados na cana-de-açúcar são caracterizados por clorose seguida de necrose, iniciando-se pelos bordos das folhas (Velini et al., 2000; Rodrigues \& Almeida, 2005). Quando utilizado em pós-emergência, os sintomas de intoxicação na cana-de-açúcar podem se apresentar de forma restrita ou ser mais acentuados nos pontos de contato da calda herbicida com as folhas (Velini et al., 2000; Rodrigues \& Almeida, 2005). A hipótese delineada nesta pesquisa é de que genótipos de cana-de-açúcar apresentam diferenciação quanto à tolerância ao herbicida inibidor do fotossistema II (FS II) e da ALS e de que esses influem de maneira direta ou indireta nas variáveis relacionadas ao uso da água pelas plantas da cultura.

Objetivou-se com este trabalho avaliar a influência dos herbicidas ametryn e trifloxysulfuron-sodium, isolados ou em mistura, sobre as características associadas ao uso da água em genótipos de cana-deaçúcar, em campo, visando selecionar aqueles mais tolerantes aos referidos herbicidas.

\section{MATERIAL E MÉTODOS}

O experimento foi realizado em campo, na Estação Experimental de Oratórios-MG, pertencente à Universidade Federal de Viçosa, em 
Tabela 1 - Análise química ${ }^{1 /}$ do solo Argissolo Vermelho-Amarelo utilizado no experimento. Oratórios-MG, 2007

\begin{tabular}{|c|c|c|c|c|c|c|c|c|}
\hline \multirow{2}{*}{\multicolumn{2}{|c|}{ Camada amostrada }} & $\mathrm{pH}$ & $\mathrm{P}$ & $\mathrm{K}^{+}$ & $\mathrm{H}+\mathrm{Al}$ & $\mathrm{Al}^{3+}$ & $\mathrm{Ca}^{2+}$ & $\mathrm{Mg}^{2-}$ \\
\hline & & $\left(\mathrm{H}_{2} \mathrm{O}\right)$ & \multicolumn{3}{|c|}{$\left(\mathrm{mg} \mathrm{dm}^{-3}\right)$} & \multicolumn{3}{|c|}{$\left(\mathrm{cmol}_{\mathrm{c}} \mathrm{dm}^{-3}\right)$} \\
\hline \multicolumn{2}{|c|}{$0-10 \mathrm{~cm}$} & 5,1 & 4,5 & 33 & 2,15 & 0,2 & 1,5 & 0,7 \\
\hline SB & CTC $(\mathrm{t})$ & CTC (T) & $\mathrm{V}$ & $\mathrm{m}$ & $\mathrm{MO}$ & Argila & Areia & Silte \\
\hline \multicolumn{3}{|c|}{$\left(\mathrm{cmol}_{\mathrm{c}} \mathrm{dm}^{-3}\right)$} & \multicolumn{2}{|c|}{$(\%)$} & \multicolumn{2}{|c|}{$\left(\right.$ dag kg $\left.^{-1}\right)$} & \multicolumn{2}{|c|}{$(\%)$} \\
\hline 2,28 & 2,48 & 2,48 & 51 & 8,0 & 0,9 & 39 & 43 & 18 \\
\hline
\end{tabular}

1/ Análise realizada no Laboratório de Análise de Solos Viçosa Ltda. pH: em água, relação 1:2,5. P-K: extrator Mehlich - 1. Ca-Mg e Al: extrator $\mathrm{KCl}-1 \mathrm{~mol} \mathrm{~L}{ }^{-1} . \mathrm{H}+\mathrm{Al}$ : extrator acetato de cálcio $0,5 \mathrm{~mol} \mathrm{~L}^{-1}-\mathrm{pH} 7,0$. SB: soma de bases trocáveis. CTC (t): capacidade de troca catiônica efetiva. CTC (T): capacidade de troca catiônica a $\mathrm{pH} 7,0$. v: saturação por bases. m: saturação por alumínio. MO: matéria orgânica $=$ C.org x 1,724 - Walkley-Black

Argissolo Vermelho-Amarelo (Embrapa, 2006). O plantio da cana-de-açúcar foi realizado em sulcos feitos após o preparo do solo, pelo sistema convencional de cultivo. A adubação foi realizada no momento do plantio, de acordo com resultados da análise do solo (Tabela 1) e recomendações para a cultura (CFSEMG, 1999). As unidades experimentais foram representadas por 10 linhas com $10 \mathrm{~m}$ de comprimento cada, espaçadas de 1,40 m. Em todos os sulcos das unidades experimentais foram depositadas 16 gemas $\mathrm{m}^{-1}$.

O delineamento experimental utilizado foi em blocos casualizados, com quatro repetições, em parcelas subdivididas. As parcelas foram constituídas pelos herbicidas ametryn $\left(2.000 \mathrm{~g} \mathrm{ha}^{-1}\right)$ e trifloxysulfuronsodium $\left(22,5 \mathrm{~g} \mathrm{ha}^{-1}\right)$, pela mistura formulada comercialmente ametryn + trifloxysulfuronsodium $\left(1.463+37,0 \mathrm{~g} \mathrm{ha}^{-1}\right)$ e uma testemunha sem aplicação de herbicidas (capinada), e as subparcelas, pelos genótipos de cana-deaçúcar RB72454, RB835486, RB855113, RB867515, RB947520 e SP80-1816.

A aplicação dos herbicidas foi realizada aos 65 dias após plantio da cana-de-açúcar, utilizando pulverizador costal pressurizado a $\mathrm{CO}_{2}$ $(254 \mathrm{kPa})$ e barra munida de quatro pontas de pulverização, modelo Teejet 110.015 , espaçadas de 0,5 m, aplicando-se o equivalente a $150 \mathrm{~L} \mathrm{ha}^{-1}$ de calda. No momento da aplicação, a temperatura situava-se em torno de $23^{\circ} \mathrm{C}$, a umidade relativa do ar era de $74 \%$ e a velocidade do vento próxima a $5 \mathrm{~km} \mathrm{~h}^{-1}$.

Decorridos 15 dias da aplicação dos herbicidas, foram realizadas as avaliações, no terço médio da primeira folha completamente expandida das plantas de cana-de-açúcar. Foi utilizado um analisador de gases no infravermelho (IRGA), marca ADC, modelo LCA $\mathrm{PRO}^{+}$ (Analytical Development Co. Ltd, Hoddesdon, UK), sendo avaliados a condutância estomática de vapores de água $\left(\mathrm{Gs}-\mathrm{mol} \mathrm{m}^{-1} \mathrm{~s}^{-1}\right)$, o gradiente entre temperatura da folha e do ar $(\Delta T)$

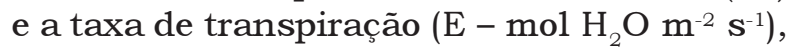
sendo calculada ainda a eficiência do uso da água (QUE - mol CO $\mathrm{mol} \mathrm{H}_{2} \mathrm{O}^{-1}$ ) a partir dos valores de quantidade de $\mathrm{CO}_{2}$ fixado pela fotossintese e quantidade de água transpirada. Após as avaliações fisiológicas, coletou-se a parte aérea da cultura, sendo posteriormente o material seco em estufa de circulação forçada de ar a $65{ }^{\circ} \mathrm{C}$, obtendo-se a massa seca da parte aérea (MSPA) por pesagem em balança analítica. Os dados foram submetidos à análise de variância pelo teste $F$, sendo efetuado posteriormente o teste de Duncan para avaliar o efeito dos tratamentos herbicidas sobre os genótipos de cana. Todos os testes foram efetuados a $5 \%$ de probabilidade de erro.

\section{RESULTADOS E DISCUSSÃO}

Observou-se para a variável massa seca das plantas de cana-de-açúcar que a testemunha apresentou maior valor quando comparada aos tratamentos herbicidas, exceto para os genótipos SP80-1816 e RB867515, quando da utilização de ametryn (Tabela 2). A sensibilidade aos herbicidas foi diferenciada entre os genótipos avaliados; com exceção do SP80-1816, todos os demais foram sensiveis aos três herbicidas aplicados, menos o 
Tabela 2 - Características fisiológicas associadas ao uso da água de genótipos de cana-de-açúcar, aos 80 dias após plantio (DAP) e 15 dias após aplicação dos herbicidas (DAA). Viçosa-MG, 2008

\begin{tabular}{|c|c|c|c|c|c|c|}
\hline \multirow{2}{*}{ Tratamento } & \multicolumn{6}{|c|}{ Genótipo } \\
\hline & RB72454 & RB835486 & RB855113 & RB867515 & RB947520 & SP80-1816 \\
\hline & \multicolumn{6}{|c|}{ Massa seca da parte aérea (g por planta) } \\
\hline Testemunha & $5,77 \mathrm{a}^{\mathrm{1} /}$ & 5,46 a & $4,93 \mathrm{a}$ & $5,02 \mathrm{a}$ & $5,02 \mathrm{a}$ & $2,46 \mathrm{a}$ \\
\hline Ametryn & $2,83 \mathrm{~b}$ & $3,69 \mathrm{ab}$ & $2,89 \mathrm{~b}$ & $5,97 \mathrm{a}$ & $3,38 \mathrm{ab}$ & $3,63 \mathrm{a}$ \\
\hline Ametryn + trifloxysulfuron-sodium & $2,90 \mathrm{~b}$ & $1,99 \mathrm{~b}$ & $2,46 \mathrm{~b}$ & $2,09 \mathrm{~b}$ & $1,66 \mathrm{~b}$ & $1,30 \mathrm{a}$ \\
\hline \multirow[t]{2}{*}{ Trifloxysulfuron-sodium } & $5,21 \mathrm{ab}$ & $2,81 \mathrm{~b}$ & $3,81 \mathrm{ab}$ & $2,99 \mathrm{~b}$ & $2,03 \mathrm{~b}$ & $2,3 \mathrm{a}$ \\
\hline & \multicolumn{6}{|c|}{ Taxa Transpiratória $-\mathrm{E}\left(\mathrm{mol} \mathrm{H}_{2} \mathrm{O} \mathrm{m}^{-2} \mathrm{~s}^{-1}\right)$} \\
\hline Testemunha & $6,91 \mathrm{~b}$ & $4,33 \mathrm{~b}$ & $5,55 \mathrm{a}$ & $6,75 \mathrm{~b}$ & $5,49 \mathrm{~b}$ & $6,92 \mathrm{a}$ \\
\hline Ametryn & $9,45 \mathrm{a}$ & 7,06 a & $7,29 \mathrm{a}$ & $9,07 \mathrm{a}$ & $10,5 \mathrm{a}$ & $6,80 \mathrm{a}$ \\
\hline Ametryn + trifloxysulfuron-sodium & $9,55 \mathrm{a}$ & $9,31 \mathrm{a}$ & $7,85 \mathrm{a}$ & $7,21 \mathrm{~b}$ & $8,46 \mathrm{ab}$ & $7,75 \mathrm{a}$ \\
\hline \multirow[t]{2}{*}{ Trifloxysulfuron-sodium } & $7,25 \mathrm{~b}$ & $7,68 \mathrm{a}$ & $7,87 \mathrm{a}$ & $7,23 \mathrm{~b}$ & $7,55 \mathrm{ab}$ & $6,79 \mathrm{a}$ \\
\hline & \multicolumn{6}{|c|}{ Condutância Estomática - Gs $\left(\mathrm{mol} \mathrm{m}^{-1} \mathrm{~s}^{-1}\right)$} \\
\hline Testemunha & $0,90 \mathrm{a}$ & $0,41 \mathrm{~b}$ & $0,49 \mathrm{~b}$ & $0,59 \mathrm{~b}$ & $0,68 \mathrm{~b}$ & $0,37 \mathrm{~b}$ \\
\hline Ametryn & $1,00 \mathrm{a}$ & $0,54 \mathrm{ab}$ & 0,95 a & $1,34 \mathrm{a}$ & $0,87 \mathrm{ab}$ & $0,44 \mathrm{~b}$ \\
\hline Ametryn + trifloxysulfuron-sodium & $0,87 \mathrm{a}$ & $0,75 \mathrm{a}$ & $0,69 \mathrm{ab}$ & $0,80 \mathrm{~b}$ & $0,97 \mathrm{a}$ & $0,74 \mathrm{a}$ \\
\hline \multirow[t]{2}{*}{ Trifloxysulfuron-sodium } & $0,43 \mathrm{~b}$ & $0,59 \mathrm{ab}$ & $0,72 \mathrm{ab}$ & $1,03 \mathrm{ab}$ & $0,49 \mathrm{c}$ & $0,56 \mathrm{ab}$ \\
\hline & \multicolumn{6}{|c|}{ Gradiente Térmico $-\Delta \mathrm{T}\left({ }^{\circ} \mathrm{C}\right)$} \\
\hline Testemunha & $2,96 \mathrm{a}$ & $2,90 \mathrm{a}$ & $2,85 \mathrm{a}$ & $2,55 \mathrm{a}$ & $2,83 \mathrm{a}$ & $2,35 \mathrm{a}$ \\
\hline Ametryn & $2,47 \mathrm{a}$ & $2,53 \mathrm{ab}$ & $1,88 \mathrm{~b}$ & $2,20 \mathrm{a}$ & $2,30 \mathrm{~b}$ & $2,32 \mathrm{a}$ \\
\hline Ametryn + trifloxysulfuron-sodium & $2,60 \mathrm{a}$ & $2,40 \mathrm{ab}$ & $2,35 \mathrm{ab}$ & $2,30 \mathrm{a}$ & $2,20 \mathrm{~b}$ & $2,70 \mathrm{a}$ \\
\hline \multirow[t]{2}{*}{ Trifloxysulfuron-sodium } & $2,30 \mathrm{a}$ & $2,00 \mathrm{~b}$ & $2,40 \mathrm{ab}$ & $2,47 \mathrm{a}$ & $2,10 \mathrm{~b}$ & $2,47 \mathrm{a}$ \\
\hline & \multicolumn{6}{|c|}{ Eficiência do Uso da Água - EUA $\left(\mathrm{mol} \mathrm{CO}_{2} \mathrm{~mol} \mathrm{H}_{2} \mathrm{O}^{-1}\right)$} \\
\hline Testemunha & $6,23 \mathrm{a}$ & 5,96 a & 7,18 a & 5,91 a & $7,38 \mathrm{a}$ & 5,56 a \\
\hline Ametryn & $4,34 \mathrm{~b}$ & $5,15 \mathrm{ab}$ & $4,87 \mathrm{~b}$ & $3,77 \mathrm{~b}$ & $4,64 \mathrm{~b}$ & $5,70 \mathrm{a}$ \\
\hline Ametryn + trifloxysulfuron-sodium & $4,68 \mathrm{ab}$ & $5,50 \mathrm{a}$ & $4,97 \mathrm{~b}$ & $4,55 \mathrm{ab}$ & $5,78 \mathrm{ab}$ & $5,92 \mathrm{a}$ \\
\hline Trifloxysulfuron-sodium & $5,10 \mathrm{ab}$ & $4,71 \mathrm{~b}$ & $5,17 \mathrm{~b}$ & $5,18 \mathrm{ab}$ & $5,85 \mathrm{ab}$ & $4,68 \mathrm{~b}$ \\
\hline
\end{tabular}

${ }^{1 /}$ Médias seguidas da mesma letra, na coluna em cada variável, não diferem pelo teste de Duncan a 5\% de probabilidade.

RB867515, o qual foi sensivel somente a trifloxysulfuron-sodium, sendo este aplicado isolado ou em mistura. Em relação à testemunha, os genótipos RB72454 e RB85513 apresentaram redução de 51,0 e 41,4\%, respectivamente, no acúmulo de massa em função da aplicação do ametryn de forma isolada ao passo que os tratados com o trifloxysulfuronsodium a redução foi de 49,6, 40,4 e 59,5\%, respectivamente para os genótipos RB835486, RB867515 e RB 947520. Em função das características morfofisiológicas diferenciadas em cada cultivar, como pilosidade, serosidade, quantidade de estômatos na folha, vigor, metabolismo, sistema radicular, porte, área foliar, entre outros, pode-se inferir a variabilidade dos resultados encontrados para a massa seca aérea das plantas. Contudo, esses fatores 
também são em grande parte responsáveis pela maior ou menor tolerância de determinados genótipos de cana-de-açúcar aos herbicidas. Esse fato já foi constatado em outros trabalhos que avaliaram a influência de herbicidas em características que compõem os componentes da produtividade de colmos de genótipos de cana-de-açúcar (Velini et al., 2000; Ferreira et al., 2005; Barela \& Christoffoleti, 2006; Azania et al., 2006).

O declínio da transpiração está associado ao fechamento dos estômatos, e as variações na abertura estomática causam alterações no potencial hídrico, por atuarem sobre a taxa transpiratória - E (Brodribb \& Hill, 2000). A planta tende a fechar os estômatos quando os níveis de luz estão abaixo da radiação fotossinteticamente ativa ou para evitar o estresse hídrico (Cochard et al., 2002). Todos esses parâmetros estão ligados numa relação de custo/benefício, pois a E também é um mecanismo de diminuir a $\Delta \mathrm{T}$. Os processos de transpiração e captura de $\mathrm{CO}_{2}$ só ocorrem quando os estômatos estão abertos, bem como a Gs. Em razão do calor latente de evaporação (o calor que efetivamente é usado para "aquecer" a água e possibilitar a evaporação), a transpiração tem um poderoso efeito resfriador importante na regulação da $\Delta \mathrm{T}$ (Farquhar \& Raschke, 1978). A E é determinada principalmente por Gs e duas variáveis físicas: radiação e déficit de saturação atmosférica (Raschke \& Zeevaart, 1976; Hunt et al., 1985).

Os resultados demonstram que a taxa de transpiração (E) dos genótipos foi influenciada pela aplicação dos herbicidas (Tabela 2). O genótipo RB72454 mostrou maior intensidade transpiratória quando sob tratamento com ametryn, isolado ou em mistura formulada com trifloxysulfuron-sodium. Por outro lado, quando tratado com o trifloxysulfuron-sodium isoladamente, observou-se efeito semelhante ao da testemunha, sendo este inferior ao dos tratados com as outras formulações. Situação semelhante foi observada para o genótipo RB867515, exceto quando este foi tratado com a mistura de herbicidas, que diferiu da aplicação de ametryn isoladamente.

A abertura e o fechamento dos estômatos são diretamente relacionados à condutância estomática de vapores de água (Gs), sendo dependentes de uma série de fatores, como radiação solar, nível de $\mathrm{CO}_{2}$ no mesofilo, umidade relativa (déficit de pressão de vapor do ar), potencial hídrico e outros de menor magnitude, como vento, substâncias de crescimento e ritmos endógenos próprios de cada espécie. A aplicação isolada de ametryn resultou em maior taxa transpiratória entre os genótipos, à exceção de RB855113 e SP80-1816, os quais não mostraram alteração dessa variável quando submetidos aos tratamentos herbicidas (Tabela 2).

A condutância foliar é composta em pequena parte pela condutância cuticular da epiderme e, quando os estômatos estão abertos, pela Gs, que é controlada pelas célulasguarda dos estômatos. Assim, a Gs é proporcional ao número e tamanho dos estômatos e diâmetro da abertura destes, características estas que dependem de outros fatores endógenos e ambientais (Brodribb \& Holbrook, 2003). De maneira geral, a condutância estomática foi maior nos tratamentos que receberam o ametryn em aplicação isolada ou em mistura formulada (Tabela 2). O genótipo RB72454 somente apresentou valores de condutância estomática inferiores aos da testemunha sob tratamento com trifloxysulfuron-sodium. Para os genótipos RB855113 e RB867515, a condutância foliar na testemunha foi inferior ao tratamento com ametryn.

$\mathrm{O}$ gradiente térmico $(\Delta \mathrm{T})$ expressa com quantos graus a temperatura da folha se encontrava acima da temperatura ambiente no momento da avaliação, podendo ser diretamente correlacionado com a intensidade metabólica do vegetal (Concenço et al., 2007a, 2009). Para os clones RB72454, RB867515 e SP80-1816, não foram observadas diferenças entre tratamentos; na média, as folhas das plantas desses genótipos apresentaram temperaturas ao redor de $2,5^{\circ} \mathrm{C}$ acima da ambiente (Tabela 2). O metabolismo do vegetal incrementa a temperatura da folha a tal ponto que, via de regra, a temperatura da folha é superior à temperatura do ar ao seu redor, que é dependente da taxa de transpiração. Assim, o aumento do metabolismo pode ser indiretamente aferido em função do gradiente entre a temperatura da folha e a do ar $(\Delta \mathrm{T})$. Normalmente essa diferença é de somente um ou dois graus, mas, em casos extremos, pode exceder $5{ }^{\circ} \mathrm{C}$ (Atkin et al., 2000; Taiz \& Zeiger, 2009). 
Uma vez que a $\Delta \mathrm{T}$ não mostrou significância estatística para os genótipos RB72454, RB867515 e SP80-1816, pode-se supor que o metabolismo não foi seriamente afetado e que esse fator provavelmente possa ser excluído da participação na Gs. As diferenças encontradas entre os genótipos devem-se às características intrinsecas deles, conforme relatado anteriormente.

Pesquisas têm relatado que os herbicidas aplicados em pós-emergência tardia da canade-açúcar demonstraram maior toxicidade em relação aos aplicados em pós-emergência inicial (Azania et al., 2006; Barela \& Christoffoleti, 2006). Em pós-emergência inicial, as plantas recuperaram-se totalmente dos efeitos tóxicos dos herbicidas, com menores reflexos na produtividade. Isso poderia ser justificado, pelo menos em parte, pelo maior número de folhas em estádio mais avançado, o que maximizaria a interceptação dos produtos. Esses autores afirmam ainda que a ocorrência de chuvas logo após a aplicação pode ter colaborado para o incremento da intoxicação em estádios mais avançados.

Todos os genótipos, exceto o SP80-1816, demonstraram, em números absolutos, temperaturas das folhas inferiores às da testemunha (estando ambos com estômatos abertos, pode indicar menor taxa de metabolismo) ao se comparar esta com os tratamentos que receberam herbicidas (Tabela 2). Para o RB835486, a planta foi mais afetada quando recebeu o trifloxysulfuron-sodium isoladamente, situando-se os demais tratamentos em posição intermediária. O inverso foi observado para RB855113, em que o tratamento com ametryn situou-se em posição inferior à observada na testemunha; os demais tratamentos herbicidas ficaram em posição intermediária. De maneira geral, o metabolismo das plantas de cana-de-açúcar foi afetado pelos herbicidas, porém não foi possivel estabelecer diferenças entre tratamentos no tocante ao potencial de inibição do metabolismo. Isso pode ter ocorrido pelo fato de a cana-de-açúcar apresentar ciclo de desenvolvimento longo, o que ocasiona recuperação de possiveis danos ao aplicar herbicidas.

De maneira geral, a eficiência de uso da água tendeu a ser semelhante entre os tratamentos herbicidas, dentro de cada genótipo (Tabela 2). Com exceção dos genótipos RB835486 e SP80-1816, ao se aplicar o trifloxysulfuron-sodium isoladamente a eficiência foi inferior à da respectiva testemunha sem aplicação. No entanto, ressalta-se que o genótipo RB855113 sobressai como um dos menos eficientes no uso da água sob aplicação dos herbicidas. O mesmo pode ser constatado para a intensidade de transpiração, em que não foram observadas diferenças entre testemunha e tratamentos herbicidas para este genótipo. Barela \& Christoffoleti (2006) destacaram que o genótipo de cana-de-açúcar RB855113 foi altamente afetado quando sob aplicação de clomazone, pendimethalin ou tebuthiuron, mas que apresentou alta capacidade de recuperação dos danos causados pelos herbicidas, de modo que esses danos na fase inicial não refletiram em redução da produtividade. Ferreira et al. (2005), ao trabalharem com 11 genótipos e quatro clones de canade-açúcar, observaram que o RB855113 foi o mais sensivel à aplicação da mistura ametryn + trifloxysulfuron-sodium.

Quando a matéria seca das plantas de cana-de-açúcar foi comparada à efíciência do uso da água, observou-se correlação entre os resultados; nos tratamentos com menor acúmulo de massa seca foi detectada também menor eficiência no uso da água, com destaque para o genótipo RB72454, em que a maior sensibilidade ao herbicida ametryn, observada pelo menor acúmulo de matéria seca, refletiu diretamente em menor eficiência de uso da água neste tratamento quando comparado à testemunha (dados não apresentados). O mesmo foi observado para as demais variáveis, em função do herbicida ao qual mais demonstram sensibilidade. A mesma relação pode ser constatada para RB72454, RB835486 e RB947520, em que a maior taxa transpiratória nos tratamentos envolvendo herbicidas acarretou menor acúmulo de matéria seca nesses mesmos tratamentos.

É possivel destacar que, de acordo com os dados, o genótipo RB855113 foi o mais sensivel aos herbicidas ametryn e trifloxysulfuronsodium, em razão dos maiores danos observados à eficiência do uso da água e transpiração. O SP80-1816 e RB867515 estiveram entre os mais tolerantes, devido à pequena alteração observada para eficiência de uso da água e gradiente térmico. 
De acordo com os resultados, pode-se concluir que as características fisiológicas condutância estomática, temperatura da folha, transpiração e eficiência de uso da água são eficientes para identificar danos de herbicidas a plantas cultivadas, principalmente aqueles com efeito sobre a taxa fotossintética, que indiretamente influencia os parâmetros associados ao uso da água. Os genótipos de canade-açúcar avaliados diferiram quanto à sensibilidade aos herbicidas aplicados, de acordo com o comportamento distinto das variáveis associadas ao uso da água sob tratamento herbicida. As variedades SP80-1816 e RB867515 destacaram-se como as menos afetadas pelos herbicidas ametryn e trifloxysulfuron-sodium, aplicados isoladamente ou em mistura, enquanto RB855113 foi a mais sensivel. Novos estudos deverão ser realizados para comparar essas variedades em função de doses dos herbicidas avaliados, a fim de possibilitar quantificação precisa do nível de suscetibilidade de cada genótipo.

\section{AGRADECIMENTOS}

Ao Conselho Nacional de Desenvolvimento Científico e Tecnológico (CNPq) e à Fundação de Amparo à Pesquisa do Estado de Minas Gerais (FAPEMIG), pelo apoio financeiro e pela concessão de bolsas.

\section{LITERATURA CITADA}

ANDREOLI, C.; SOUZA, S. P. Cana-de-açúcar: a melhor alternativa para conversão da energia solar e fóssil em etanol. Econ. Energia, v. 59, n. 2, p. 27-33, 2006

ATKIN, O. K. et al. Leaf respiration of snow gum in the light and dark. Interactions between Temperature and Irradiance. Plant Physiol., v. 122, n. 3, p. 915-923, 2000.

AZANIA, C. A. M. et al. Seletividade de herbicidas: III aplicação de herbicidas em pós-emergência inicial e tardia da cana-de-açúcar na época da estiagem. Planta Daninha, v. 24, n. 3, p. 489-495, 2006.

BARELA, J. F.; CHRISTOFFOLETI, P. J. Seletividade de herbicidas aplicados em pré-emergência da cultura da cana-deaçúcar (RB 867515) tratada com nematicidas.

Planta Daninha, v. 24, n. 2, p. 371-378, 2006.

BAPTISTA, J. M. et al. Programa nacional para o uso eficiente da água. Lisboa: Instituto Superior de Agronomia, 2001. 212 p.
BRODRIBB, T. J.; HILL, R. S. Increases in water potential gradient reduce xylem conductivity in whole plants. Evidence from a low-pressure conductivity method. Plant Physiol., v. 123, n. 3, p. 1021-1028, 2000.

BRODRIBB, T. J.; HOLBROOK, N. M. Stomatal closure during leaf dehydration, correlation with other leaf physiological traits. Plant Physiol., v. 132, n. 4, p. 21662173, 2003 .

COMISSÃO DE FERTILIDADE DO SOLO DO ESTADO DE MINAS GERAIS - CFSEMG -. Recomendações para o uso de corretivos e fertilizantes em Minas Gerais - 5 aproximação. Viçosa, MG: Universidade Federal de Viçosa, 1999. 359 p.

CONCENÇO, G. et al. Uso da água em biótipos de azevém (Lolium multiflorum) em condição de competição. Planta Daninha, v. 25, n. 3, p. 449-455, 2007a.

CONCENÇO, G. et al. Plasmodesmos: transporte simplástico de herbicidas na planta. Planta Daninha, v. 25, n. 2, p. 423-432, 2007b.

CONCENÇO, G. et al. Uso da água por plantas de arroz em competição com biótipos de Echinochloa crusgalli resistente e suscetível ao herbicida quinclorac. Planta Daninha, v. 27, n. 2, p. 249-256, 2009.

COCHARD, H. et al. Unraveling the effects of plant hydraulics on stomatal closure during water stress in walnut. Plant Physiol., v. 128, n. 1, p. 282-290, 2002.

EMPRESA BRASILEIRA DE PESQUISA AGROPECUÁRIA - EMBRAPA. Centro Nacional de Pesquisa Agropecuária de Solos. Sistema brasileiro de classificação de solos. Rio de Janeiro: 2006. 412 p.

FARQUHAR, G. D.; RASCHKE, K. On the resistance to transpiration of the sites of evaporation within the leaf. Plant Physiol., v. 61, n. 6, p. 1000-1005, 1978.

FERREIRA, E. A. et al. Sensibilidade de cultivares de canade-açúcar à mistura trifloxysulfuron-sodium + ametryn. Planta Daninha, v. 23, n. 1, p. 93-99, 2005

HUNT, E. R. et al. Effects of nitrate application on Amaranthus powellii Wats II. Stomatal response to vapor pressure difference is consistent with optimization of stomatal conductance. Plant Physiol., v. 79, n. 3, p. 614-618, 1985.

PEREIRA-NETTO, A. B. Crescimento e desenvolvimento. In: WACHOWICZ, C. M.; CARVALHO, R. I. N. (Eds.). Fisiologia vegetal - produção e pós-colheita. Curitiba: Champagnat, 2002. p. 17-42.

PROCÓPIO, S. O. et al. Características fisiológicas das culturas de soja e feijão e de três espécies de plantas daninhas. Planta Daninha, v. 22, n. 2, p. 211-216, 2004. 
RASCHKE, K.; ZEEVAART, J. A. D. Abscisic acid content, transpiration, and stomatal conductance as related to leaf age in plants of Xanthium strumarium L. Plant Physiol., v. 58, n. 2, p. 169-174, 1976.

RODRIGUES, B.N.; ALMEIDA, F. S. Guia de herbicidas 5.ed. Londrina, 2005. 592 p.

TAIZ, L.; ZEIGER, E. Fisiologia Vegetal. 4.ed., Porto Alegre: Artmed, 2009. 848 p.
VELINI, E. D. et al. Avaliação da seletividade da mistura de oxyfluorfen e ametryne, aplicada em pré e pós-emergência, a dez variedades de cana-de-açúcar (cana planta).

Planta Daninha, v. 18, n. 2, p. 123-134, 2000.

VIVIAN, R. et al. Persistência e lixiviação de ametryn e trifloxysulfuron-sodium em solo cultivado com cana-de-açúcar. Planta Daninha, v. 25, n. 1 p. 111-124, 2007. 